\title{
Establishment of Human Neural Progenitor Cells from Human Induced Pluripotent Stem Cells with Diverse Tissue Origins
}

\author{
Hayato Fukusumi, ${ }^{1}$ Tomoko Shofuda, ${ }^{2}$ Yohei Bamba, ${ }^{1,3}$ Atsuyo Yamamoto, \\ Daisuke Kanematsu, ${ }^{1}$ Yukako Handa, ${ }^{1}$ Keisuke Okita, ${ }^{4}$ Masaya Nakamura, ${ }^{5}$ \\ Shinya Yamanaka, ${ }^{4}$ Hideyuki Okano, ${ }^{3}$ and Yonehiro Kanemura ${ }^{1,6}$ \\ ${ }^{1}$ Division of Regenerative Medicine, Institute for Clinical Research, Osaka National Hospital, National Hospital Organization, \\ Osaka 540-0006, Japan \\ ${ }^{2}$ Division of Stem Cell Research, Institute for Clinical Research, Osaka National Hospital, National Hospital Organization, \\ Osaka 540-0006, Japan \\ ${ }^{3}$ Department of Physiology, Keio University School of Medicine, Tokyo 160-8582, Japan \\ ${ }^{4}$ Center for iPS Cell Research and Application (CiRA), Kyoto University, Kyoto 606-8507, Japan \\ ${ }^{5}$ Department of Orthopedic Surgery, Keio University School of Medicine, Tokyo 160-8582, Japan \\ ${ }^{6}$ Department of Neurosurgery, Osaka National Hospital, National Hospital Organization, Osaka 540-0006, Japan \\ Correspondence should be addressed to Yonehiro Kanemura; kanemura@onh.go.jp
}

Received 24 December 2015; Accepted 28 March 2016

Academic Editor: Kaylene Young

Copyright (C) 2016 Hayato Fukusumi et al. This is an open access article distributed under the Creative Commons Attribution License, which permits unrestricted use, distribution, and reproduction in any medium, provided the original work is properly cited.

Human neural progenitor cells (hNPCs) have previously been generated from limited numbers of human induced pluripotent stem cell (hiPSC) clones. Here, 21 hiPSC clones derived from human dermal fibroblasts, cord blood cells, and peripheral blood mononuclear cells were differentiated using two neural induction methods, an embryoid body (EB) formation-based method and an EB formation method using dual SMAD inhibitors (dSMADi). Our results showed that expandable hNPCs could be generated from hiPSC clones with diverse somatic tissue origins. The established hNPCs exhibited a mid/hindbrain-type neural identity and uniform expression of neural progenitor genes.

\section{Introduction}

Human neural progenitor cells (hNPCs), which are present in fetal and adult neural tissues, have the potential to be therapeutically beneficial in the treatment of neuronal diseases such as spinal cord injury or stroke; however, it is technically difficult to obtain hNPCs from human neural tissues. The development of human embryonic stem cells (hESCs) [1] and human induced pluripotent stem cells (hiPSCs) $[2,3]$ has greatly improved the prospects of regenerative medicine. We are now able to obtain unlimited hiPSCs from every somatic tissue source [4]. However, hiPSC clones exhibit variable differentiation propensities [5], similar to hESCs [6].

Many protocols have been reported for the neural induction of hESCs/hiPSCs. Transplantable neural precursors were first derived from hESCs, which were subjected to spontaneous embryoid body (EB) formation, followed by neural rosette selection [7]. EB-mediated neural rosette formation is used not only for establishing rosette-stage neural stem cells (R-NSCs) from hESCs [8], but also for generating longterm self-renewing neuroepithelial-like stem (lt-NES) cells from hESCs/hiPSCs $[9,10]$. However, the neural induction efficiency of these methods depends on the innate differentiation propensity of the hESC/hiPSC clones [11]. Using a strategy based on the neural default model, inhibitors of the bone morphogenic protein (BMP) signaling pathway, such as Noggin or the small molecule Dorsomorphin, have been used to direct the differentiation of hESCs/hiPSCs toward the neural lineage [12]. In addition, Lefty-A or the small molecule SB431542 can be used to inhibit Nodal, a member 
of the transforming growth factor (TGF) $\beta$ family which contributes to endodermal or mesodermal fate selection, promoting neural induction of hESCs. The combination of a BMP antagonist and a TGF $\beta /$ Activin/Nodal inhibitor has been used to accelerate the neural induction of hESCs/hiPSCs [11, 13-15]. To establish a reproducible EB-based method, Eiraku et al. subjected fully dissociated hESCs/hiPSCs to SFEBq (serum-free culture of EB-like aggregates) in the presence of a Rho-associated protein kinase (ROCK) inhibitor, resulting in the formation of uniformly sized EBs [16]. It has also been shown that neural precursor cells can be derived from hESCs at physiological oxygen levels (3 to 5\%) [17].

Neural induction using these methods has been successful for only a limited number of hESC/hiPSC clones. Koyanagi-Aoi et al. recently reported on the SFEBq-mediated induction of dopaminergic neurons from hESCs and hiPSCs derived from various somatic tissues [18]. The aim of the current study was to determine whether hNPCs can be derived from any hiPSC clone regardless of its somatic tissue origin. We evaluated 21 hiPSC clones derived from human dermal fibroblasts (HDFs, 13 clones), cord blood (CB) cells (3 clones), and peripheral blood mononuclear (PBMN) cells (5 clones) using an EB formation-based method (EBFM) and an EB formation method that includes dual SMAD inhibitors (dSMADi). Although there is consensus that SMAD inhibition is necessary for neural induction as mentioned above, there are many variations among methods. Therefore, we performed the dSMADi method, in which the conditions are easily controlled, using two types of media and two different oxygen levels. Thus, the previously reported neural induction method was expanded into four conditions. Our data indicate that dual SMAD inhibition can be used to generate mid/hindbrain-type hNPCs from hiPSCs regardless of their somatic tissue origin. These expandable hNPCs may be a useful cell source for regenerative medicine research and the treatment of neuronal diseases.

\section{Materials and Methods}

2.1. Culture of hiPSCs. This study was conducted in accordance with the principles of the Helsinki Declaration, and the use of hiPSC clones was approved by the ethics committee of Osaka National Hospital (number 110) and CiRA, Kyoto University. All hiPSC clones (Table S1 in Supplementary Material available online at http://dx.doi.org/10.1155/2016/7235757) were cultured at the CiRA on mitomycin C-treated SNL feeder cells in primate ES cell medium (ReproCELL) until $\sim 50 \%$ confluent and then transported to our laboratory at Osaka National Hospital. The hiPSC clones were cultured for two days before neural induction.

2.2. Neural Induction of hiPSCs. Two neural induction methods were used in this study: EBFM $[19,20]$ and an EB formation method using dual SMAD inhibitors (dSMADi) [14, 16]. Each of the hiPSC clones was simultaneously subjected to neural induction using the two methods.
For EBFM, the hiPSCs were treated with $10 \mu \mathrm{M}$ Y-27632 (ROCK inhibitor) for $1 \mathrm{~h}$ at $37^{\circ} \mathrm{C}$ and then detached using $1 \mathrm{mg} / \mathrm{mL}$ collagenase IV (Life Technologies) and floated onto gelatin-coated dishes to remove the SNL feeder cells. After 30 min, floating EBs were transferred to Petri dishes containing DMEM/F12 (D6421, Sigma) with 20\% knockout serum replacement (KSR, Life Technologies), $0.1 \mathrm{mM}$ nonessential amino acids (NEAA, Life Technologies), $2 \mathrm{mM} \mathrm{L}$-glutamine (Life Technologies), $0.1 \mathrm{mM}$ 2-Mercaptoethanol (2-ME, Life Technologies), antibiotic-antimycotic (Life Technologies), and $10 \mu \mathrm{M}$ Y-27632 (day 0). The next day (day 1), the medium was replaced with 5\% KSR-containing medium and cultured further for 30 days with a medium change every two days.

For dSMADi, hiPSCs were treated with $10 \mu \mathrm{M} \mathrm{Y-27632}$ for $1 \mathrm{~h}$ at $37^{\circ} \mathrm{C}$ and then dissociated with Trypsin/EDTA to generate single-cell suspensions and suspended in two types of medium: KSR-based medium [DMEM/F12 (D6421) with $20 \%$ KSR, $0.1 \mathrm{mM}$ 2-ME, $10 \mu \mathrm{M}$ SB-431542 (SB, Sigma), and $2 \mu \mathrm{M}$ Dorsomorphin (DSM, Wako)] and B27N2-based medium [DMEM/F12 (D8062) with $15 \mathrm{mM}$ HEPES, 5\% B27, $5 \%$ N2 supplement (N2, Life Technologies), $10 \mu \mathrm{M}$ SB, $2 \mu \mathrm{M}$ $\mathrm{DSM}$, and $10 \mathrm{ng} / \mathrm{mL} \mathrm{bFGF}$. Both media were supplemented with $30 \mu \mathrm{M}$ Y-27632 for the first 3 days. Completely dissociated cells were then seeded into ultralow attachment 96well plates (PrimeSurface ${ }^{\circledR}$ 96-well, Sumitomo Bakelite) at 9,000 cells/well, centrifuged at $700 \mathrm{rpm}$ for $3 \mathrm{~min}$ (quickaggregation), and cultured in a $5 \% \mathrm{CO}_{2}$ incubator with 5 or $20 \% \mathrm{O}_{2}$. Thus, the dSMADi neural induction was conducted using four conditions: $\mathrm{KSR} / 20 \% \mathrm{O}_{2}, \mathrm{KSR} / 5 \%$ $\mathrm{O}_{2}, \mathrm{~B} 27 \mathrm{~N} 2 / 20 \% \mathrm{O}_{2}$, and $\mathrm{B} 27 \mathrm{~N} 2 / 5 \% \mathrm{O}_{2}$. The cells were cultured for 14 days with daily replacement of half the spent medium with fresh medium. On day 14, the aggregates were dissociated mechanically and cultured on Petri dishes in a $5 \% \mathrm{CO}_{2}$ incubator with $20 \% \mathrm{O}_{2}$ to generate the first passage of hNPCs. Neurospheres were generated from the second passage of NPCs by completely dissociating the cells with Accutase $^{\mathrm{TM}}$ (Innovative Cell Technologies) and then cultured on nontreated flasks. If the hNPCs attached to the culture vessels at early passages, we used ultralow attachment dishes (PrimeSurface, Sumitomo Bakelite) to establish the hNPCs as neurospheres.

2.3. Maintenance of hNPCs. The hNPCs were seeded at 1 $\times 10^{5}$ cells $/ \mathrm{mL}$ and cultured as floating neurospheres in hNPC medium [DMEM/F12 (D8062) with $15 \mathrm{mM}$ HEPES, 2\% B27, 20 ng/mL EGF (PeproTech), 20 ng/mL FGF2 (PeproTech), $10 \mathrm{ng} / \mathrm{mL}$ leukemia inhibitory factor (Millipore), and $5 \mu \mathrm{g} / \mathrm{mL}$ heparin (Sigma-Aldrich)].

\subsection{Quantitative Reverse Transcription-Polymerase Chain} Reaction (Quantitative RT-PCR). Total RNA was extracted using the RNeasy MinElute Cleaning Kit (Qiagen), and the cDNAs were synthesized using the PrimeScript ${ }^{\circledR}$ RT Master Mix (Takara Bio) according to the manufacturer's specifications. Quantitative PCR analysis was performed using genespecific primers (Table S4), the Power SYBR ${ }^{\circledR}$ Green PCR Master Mix, and the 7300 Real-Time PCR System (Applied Biosystems). Gene expression levels were expressed as delta $\mathrm{Ct}$ values normalized to GAPDH [23]. 
2.5. Measurement of Neural Aggregate Size. Phase-contrast images of eight wells per condition for each clone were captured (one representative image per condition is shown in Figure S3). The projected areas of the neural aggregates were measured using ImageJ [24]. The aggregate size was calculated as a sphere volume using the circular diameter determined from the projected area [25].

2.6. In Vitro Neuronal Differentiation. To avoid disturbing the naturally formed niche, the neurospheres were not dissociated. The intact neurospheres were transferred to vessels coated with Growth Factor Reduced Matrigel ${ }^{\mathrm{TM}}$ (diluted to $1: 30, \mathrm{BD}$ Biosciences) and cultured in Neurobasal Medium (Life Technologies) containing 2\% B27 and 1\% L-glutamine for 2 weeks [22].

2.7. Immunocytochemical Staining. Cells were fixed in $4 \%$ paraformaldehyde and washed with PBS. The fixed samples were then blocked with $10 \%$ normal goat serum and incubated with anti- $\beta$ III tubulin antibody (clone TuJ1, Babco) overnight at $4^{\circ} \mathrm{C}$. The samples were then incubated with AlexaFluor-488-conjugated goat anti-mouse IgG (Molecular Probes, Life Technologies) for $1 \mathrm{~h}$ at room temperature. The stained samples were examined with a confocal laserscanning microscope. All staining procedures were performed with matched-isotype controls [22].

2.8. Neurite Analysis. $\beta$ III tubulin-positive neurites in four regions were detected by appropriate thresholding and then skeletonized using ImageJ [24]. Total neurite length was determined by counting the positive pixels [26].

2.9. Statistical Analysis. Significant differences in gene expression levels obtained by quantitative RT-PCR were analyzed using the Steel-Dwass nonparametric multiple comparison test or Welch's $t$-test. Significant differences in neural aggregate size were also analyzed by the Steel-Dwass comparison test. Significant differences in total neurite length were analyzed by Dunnett's test. See figure legends for details.

\section{Results and Discussion}

There are many variations among neural induction methods, although there is consensus about the necessity of SMAD inhibition. To determine whether hiPSC clones derived from different somatic tissues could differentiate into hNPCs without specific neural induction methods (Figure 1(a)), we examined 21 hiPSC clones established in CiRA (Table S1). These hiPSC clones were derived from three different tissues: HDFs, CB cells, and PBMN cells (Table S1).

All of the hiPSCs exhibited a typical undifferentiated hESC-like morphology (Figure S1A). Quantitative RT-PCR showed that the clones expressed uniformly high levels of the pluripotency marker genes Oct4, NANOG, and LIN28A but very low levels of the differentiation marker genes SOX17 (endoderm), $T$ (mesoderm), and SOX1 and PAX6 (both neural) just prior to neural induction (Figure 1(b) and Figure S1B).
Two predominant methods for inducing the neural differentiation of hESCs/hiPSCs are the EB formationbased method (EBFM) and EB formation with dual SMAD inhibitors (dSMADi). We controlled the aggregate size in the dSMADi method using a quick-aggregation procedure [16] and examined four additional conditions using this method by assessing combinations of two culture media and two different oxygen levels. In the EBFM approach, we used a low concentration (5\%) of knockout serum replacement (KSR) $[19,20]$ to limit the amount of BMP-like activity [27], which opposes neural induction and is present in the KSR.

We subjected the 21 hiPSC clones to the five different neural induction procedures (Figure 1(a)). To assess the neural induction efficiency using dSMADi, we compared gene expression levels among the hiPSCs, day 30 EBFMderived EBs, and day 14 dSMADi-derived aggregates, by quantitative RT-PCR (Figure 1(b), Figure S1B, Figure S1C, and Figure S2). Bivariate box plots showed that the pluripotency marker genes, Oct4 and NANOG, were strongly and uniformly downregulated in day $14 \mathrm{dSMADi}$-derived aggregates but not in day 30 EBFM-derived EBs, which exhibited more variable expression among the clones (Figure 1(b) and Figure $\mathrm{S1C})$. Interestingly, dSMADi treatment also resulted in the slight downregulation of another pluripotency marker gene, LIN28A (Figure 1(b)).

Day 30 EBs did not uniformly express the endoderm marker gene SOX17, mesoderm marker gene $T$, or neural marker genes SOX1 and PAX6 and were classified as nonneural, neural, or three germ layer-containing EBs (Figure S1D). In contrast, almost all of the day 14 dSMADi-derived aggregates exhibited the upregulation of both neural marker genes but not nonneural marker genes (Figure 1(b)). These findings indicated that while EBFM-derived EBs exhibited cell lineage variability (only 6 clones differentiated toward specifically neural lineage), dSMADi-derived aggregates exhibited less clonal variation, and almost all of them underwent neural lineage induction regardless of somatic tissue origin and the differentiation protocols (over $90 \%$ of clones differentiated toward neural lineage). Hereafter, we will refer to day 14 dSMADi aggregates as neural aggregates.

We further evaluated the effects of the four dSMADi conditions by comparing the gene expression levels of the neural aggregates derived in two types of media (KSR or B27N2) and two different oxygen levels (20\% or $5 \%$ ) (Figure 2(a)). Although the expression levels of the pluripotency marker gene Oct4 and neural marker gene SOX1 were similar in aggregates cultured in the four conditions, those of NANOG in aggregates cultured in $\mathrm{KSR} / 5 \% \mathrm{O}_{2}$ and of PAX6 in aggregates cultured in $\mathrm{KSR} / 20 \% \mathrm{O}_{2}$ and $\mathrm{KSR} / 5 \% \mathrm{O}_{2}$ were significantly higher than in the B27N2-based conditions (Figure 2(a)). The oxygen level alone did not significantly impact the expression levels of these genes. Although we used a quick-aggregation procedure in the dSMADi experiments to eliminate the size and shape variability observed in EBFMderived EBs (Figure S1A), we noticed obvious differences in neural aggregate size among the four conditions (Figure S3).

To examine the effects of the culture conditions in detail, we measured the size of the neural aggregates on day 7 and day 14 (Figure 2(b)). The day 14 neural aggregates cultured in 


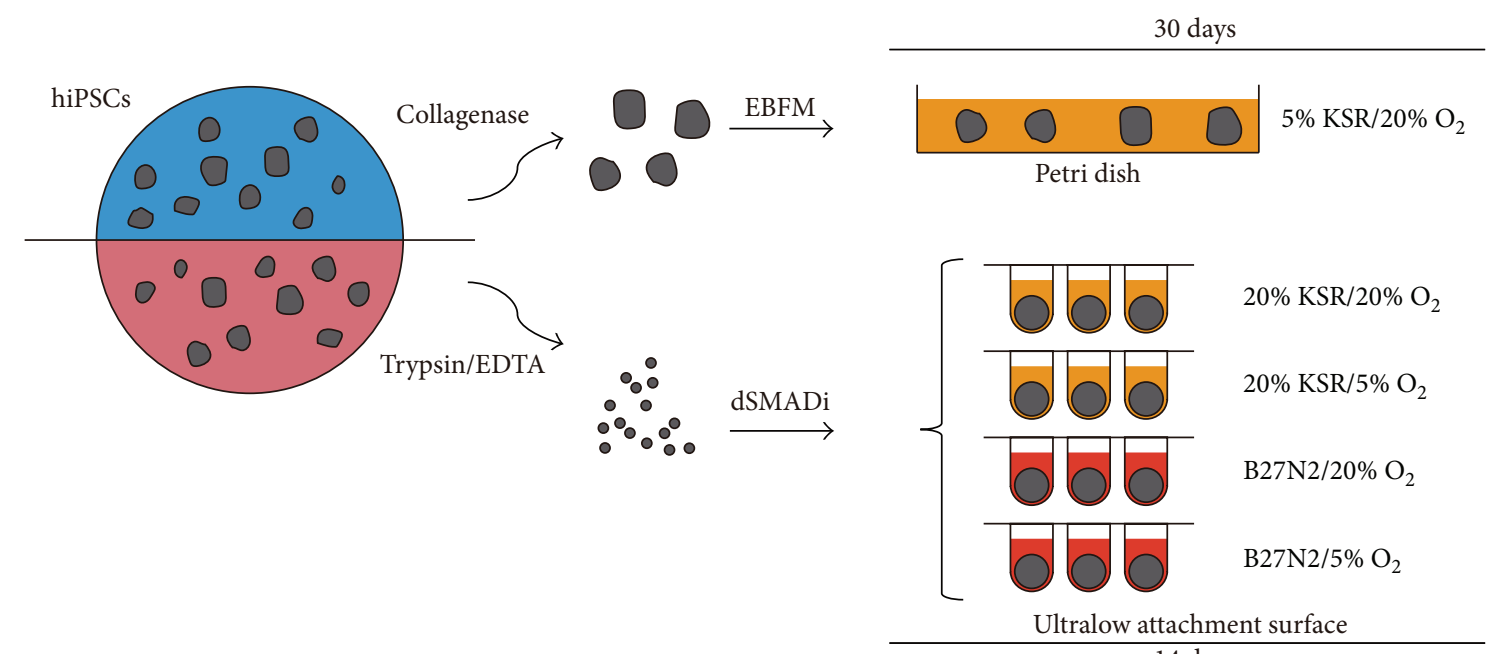

(a)
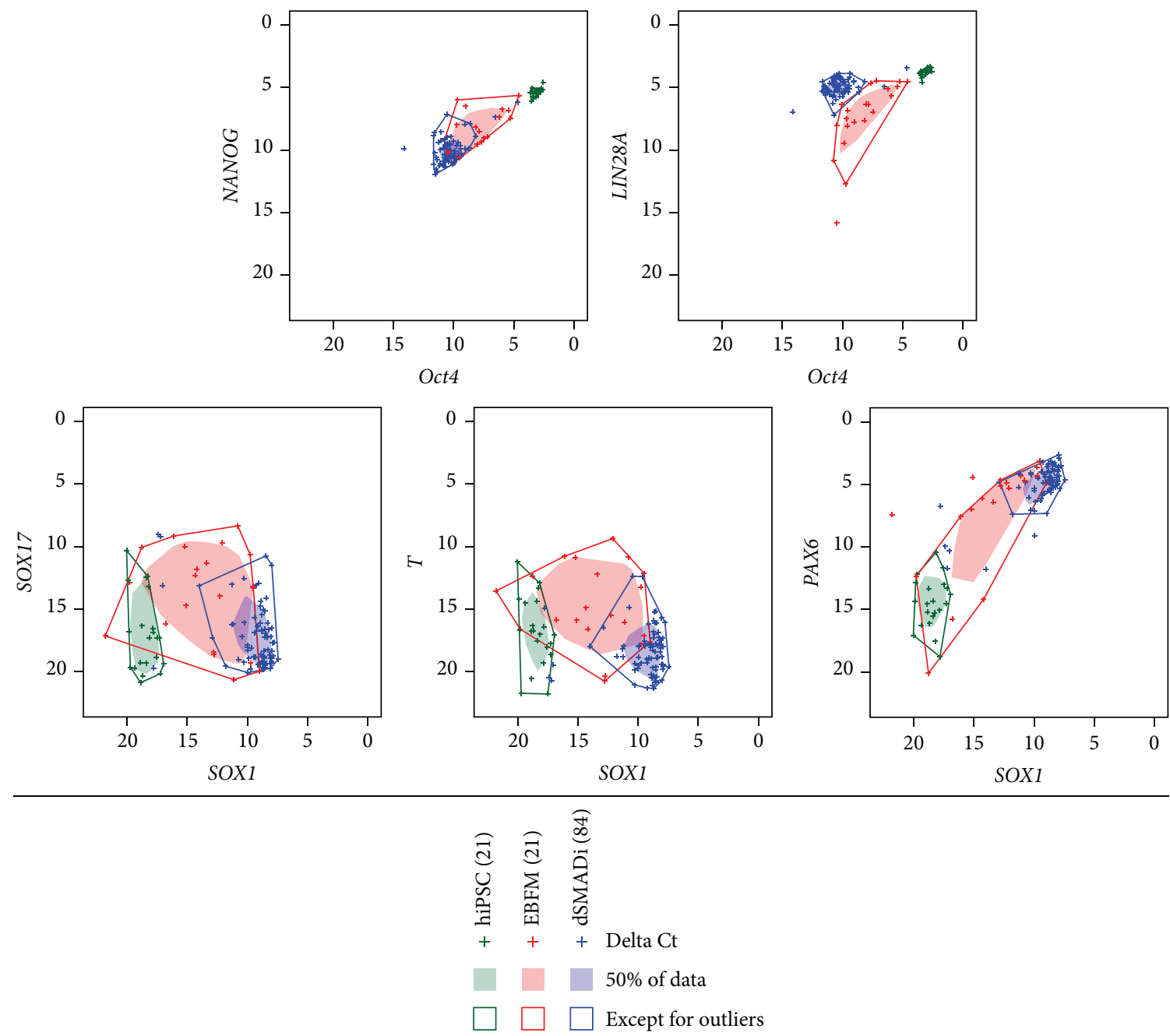

(b)

FIGURE 1: dSMADi improves the neural induction efficiency of hiPSCs regardless of their somatic tissue origin. (a) Schematic drawing of the neural induction methods used in this study. (b) Bivariate box plots displaying gene expression levels in hiPSCs, day 30 EBFM-derived EBs, and day 14 dSMADi-derived aggregates. Quantitative RT-PCR-generated delta Ct values for the pluripotency marker genes (Oct4, NANOG, and LIN28A), endoderm marker gene (SOX17), mesoderm marker gene (T), and neural marker genes (SOX1 and PAX6) are shown. Green: hiPSCs; red: EBFM; blue: dSMADi. The numbers of clones analyzed are indicated in parentheses and "+" symbols represent each of the delta Ct values. Filled-in regions contain the $50 \%$ of the data points, and data points outside of the surrounding lines represent outliers. See also Figures S1 and S2. 

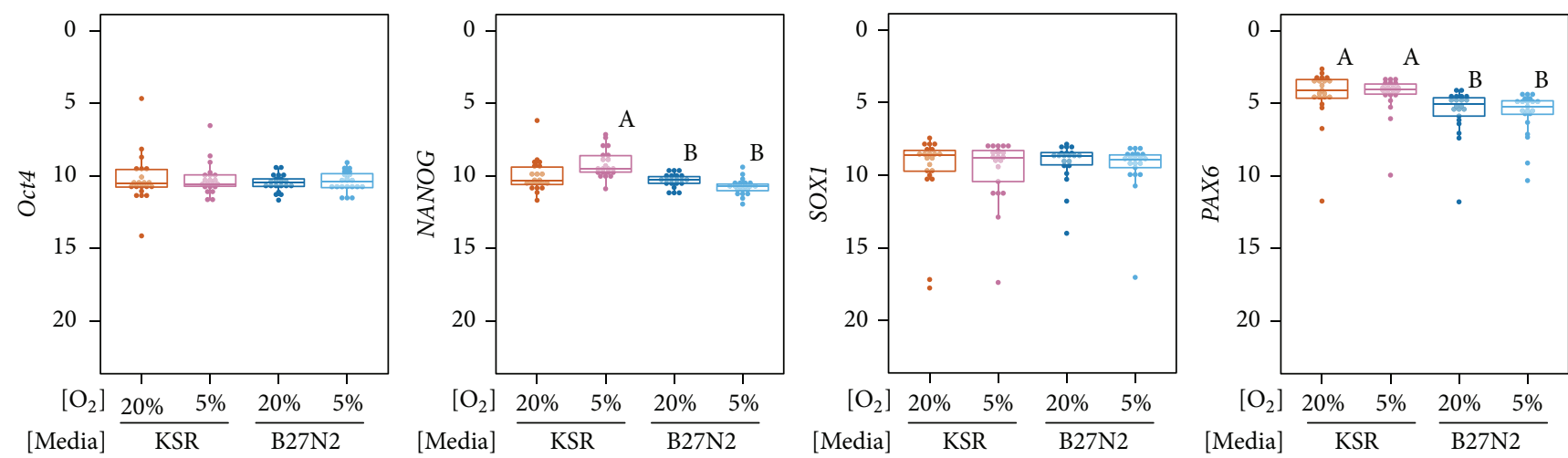

(a)

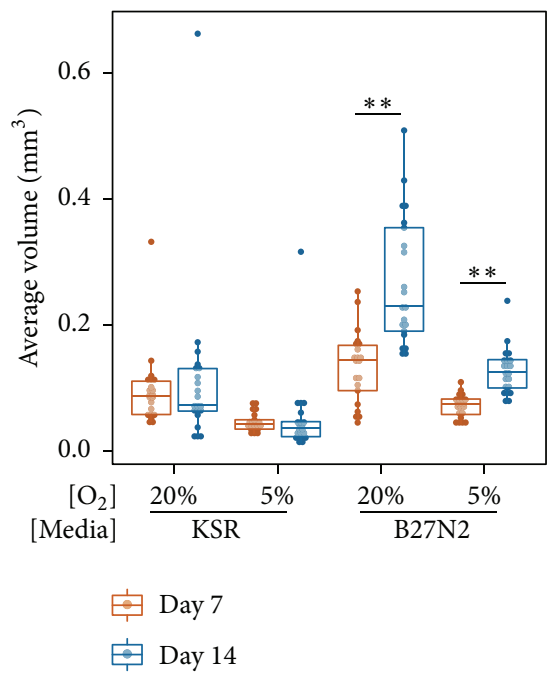

(b)

Figure 2: Effects of media and oxygen level on neural aggregate gene expression and size. (a) Dot plots and box plots showing for the gene expression levels of pluripotency markers (Oct4 and NANOG) and neural markers (SOX1 and PAX6) in day 14 aggregates cultured in the various conditions. Twenty-one clones were analyzed in each condition. Statistical significance was determined by the Steel-Dwass test. Statistically significant differences $(p<0.01)$ were found between samples "A" and "B". See also Figure S2. (b) Dot plot and box plot showing the size of day 7 and day 14 neural aggregates. Statistical analysis was performed with the Steel-Dwass test $\left({ }^{* *} p<0.01\right)$. All $p$ values are shown in Table S2.

B27N2-based conditions were significantly larger than those cultured in KSR-based conditions at each oxygen level, and they were also significantly larger when cultured in $20 \% \mathrm{O}_{2}$ than in $5 \% \mathrm{O}_{2}$, in both types of media (Figure 2(b) and Table S2). Notably, neural aggregates did not grow in the KSR-based conditions over the induction period regardless of the oxygen level. In contrast, B27N2, which contains basic fibroblast growth factor, supported the growth of the neural aggregates (Figure 2(b)). These findings indicated that although the four dSMADi conditions efficiently promoted neural lineage induction, the culture medium influenced both gene expression and aggregate size, whereas the oxygen level primarily affected aggregate size.

Next, we investigated whether the neural aggregates exhibited a forebrain-type property, as previously observed for lt-NES cells and R-NSCs [8-10]. Because the expression of PAX6 was higher in KSR-derived aggregates than in B27N2-derived aggregates, we first compared the expression levels of the forebrain marker genes, FOXG1 and OTX1, in day 14 neural aggregates cultured in all four conditions. Although there were no significant differences in FOXG1 and OTX1 expression levels among aggregates cultured in the different conditions (Figure 3(a)), the FOXG1 expression was more variable among the clones compared to the OTX1 expression. Therefore, we compared the FOXG1 expression among aggregates derived from somatic tissues of different origins (Figure 3(b)). Notably, the FOXG1 expression was significantly lower in the clones derived from PBMN cells than in those derived from HDFs and CB cells (Figure 3(b)), although the HDF- and CB cell-derived clones exhibited variable FOXG1 expression. Given that ES cells generate anterior forebrain-like neural precursor cells in the absence of external signals [28], the variability of FOXG1 expression in the HDF- and CB-derived clones might reflect variable Wnt activation in the hiPSCs [29]. 

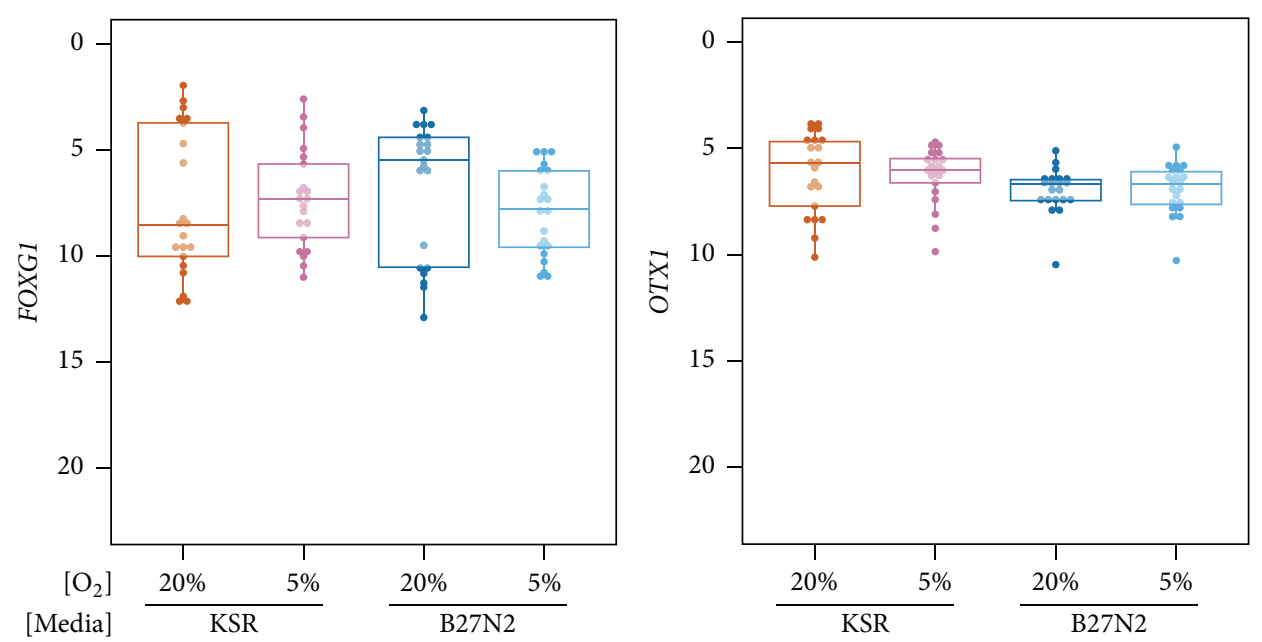

(a)
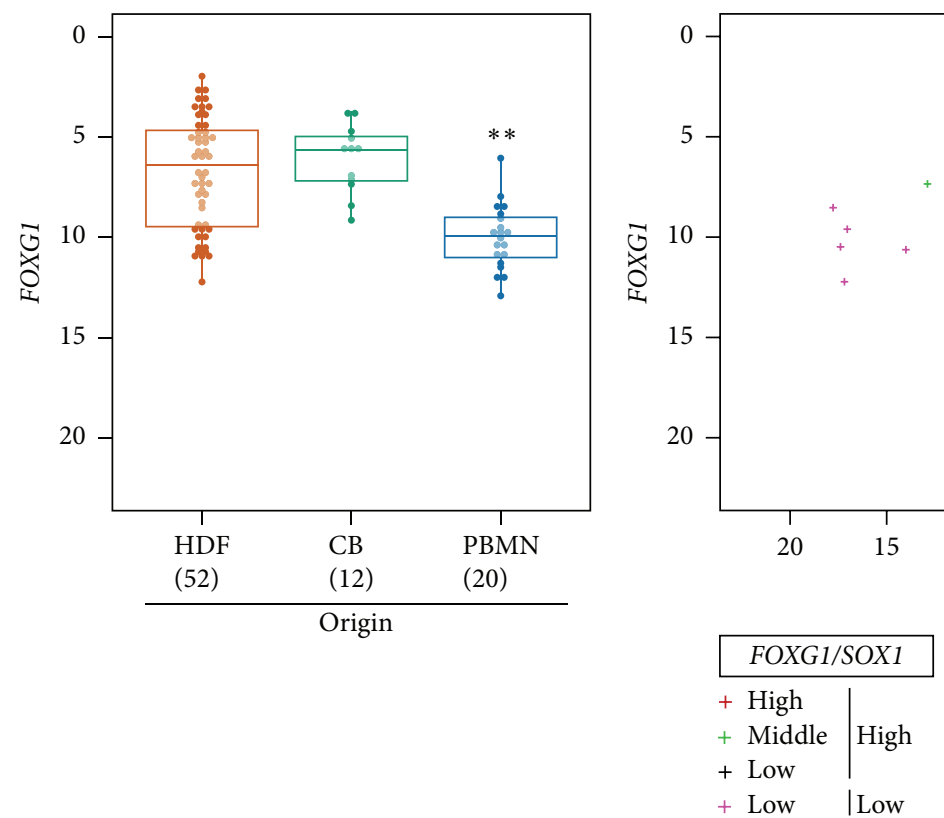

(b)

(c)

FIGURE 3: PBMN-derived neural aggregates exhibit low FOXG1 expression levels. (a) Dot plots and box plots showing FOXG1 and OTX1 expression in day 14 neural aggregates cultured in the various conditions. (b) Dot plots and box plots showing the FOXG1 expression levels according to tissue origin. The number of clones analyzed is indicated in parentheses. Statistical analysis was performed by the Steel-Dwass test. ${ }^{* *} p<0.01$. (c) Clustering of clones, based on the expression of FOXG1 and SOX1, using the K-medoids method. The delta Ct values are indicated by “+” symbols. Four clusters were identified: FOXG1-high/SOX1-high (red), FOXG1-middle/SOX1-high (green), FOXG1-low/SOX1high (black), and FOXG1-low/SOX1-low (pink). See also Table S3.

However, the mechanisms regulating differential FOXG1 expression between CB- and PBMN-derived clones are unclear because both hiPSC clones were derived from mesodermal tissues, cultured for over 20 passages, and were considered to have lost their somatic tissue-specific epigenetic memory [30-32]. Given that all of the PBMN cells were $\alpha \beta \mathrm{T}$ cells in this study, their genomes were modified due to T-cell receptor (TCR) rearrangement, in contrast to the HDF and CB cells. The genes encoding T-cell receptor alpha (TRA) and T-cell receptor beta (TRB) are located at chromosomal regions 14q11.2 and 7q34, respectively. FOXG1 is located at chromosomal region 14q13. Although TRA and FOXG1 are separated by about 6 million bases, some epigenetic modifications might still occur in the regulatory region of FOXG1. FOXG1 is critical for normal corticogenesis [33]; therefore, PBMN-derived hiPSCs might not be a good source for analyzing normal cortical development or disease modeling. Further studies are required to elucidate whether inhibition of Wnt signaling improves the induction of anterior neural progenitors from PBMN-derived clones. On the other hand, we hypothesized that neural aggregates with the potential for producing uniform hNPCs could be 

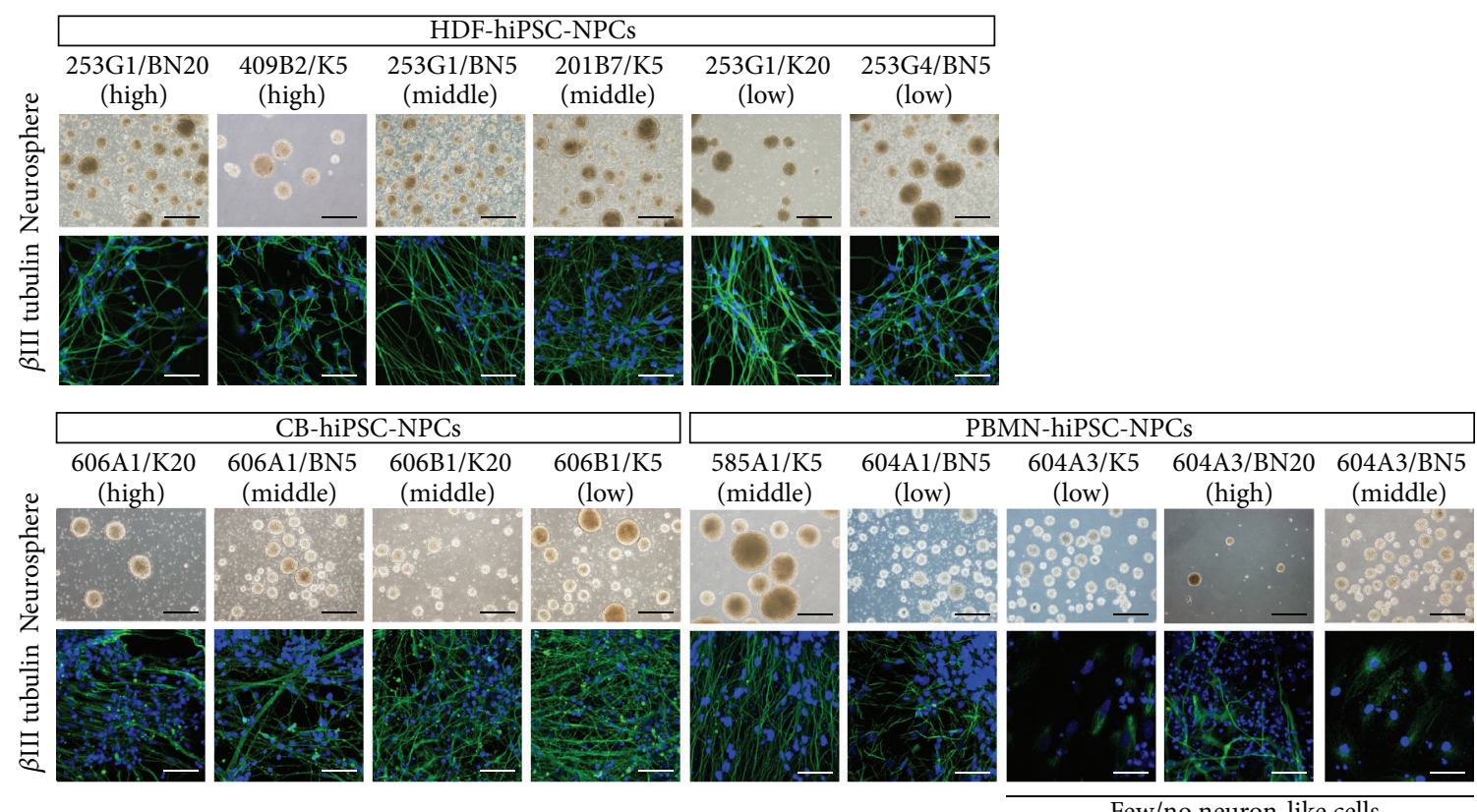

(a)
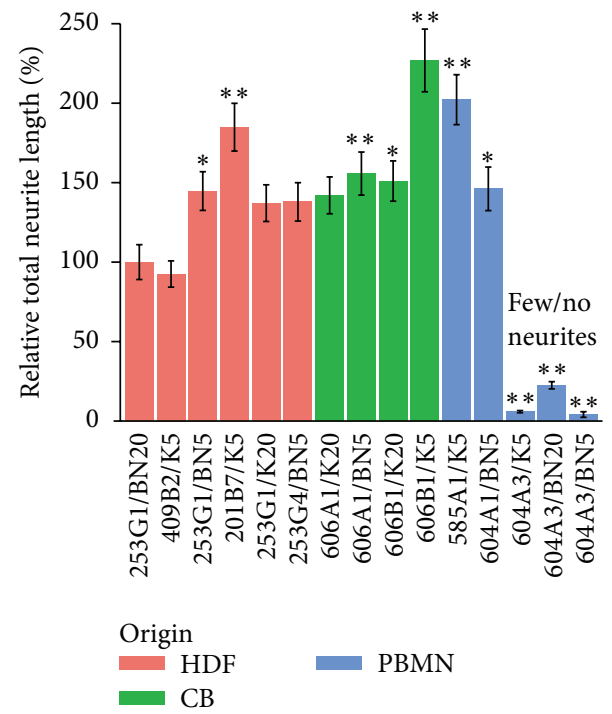

(b)

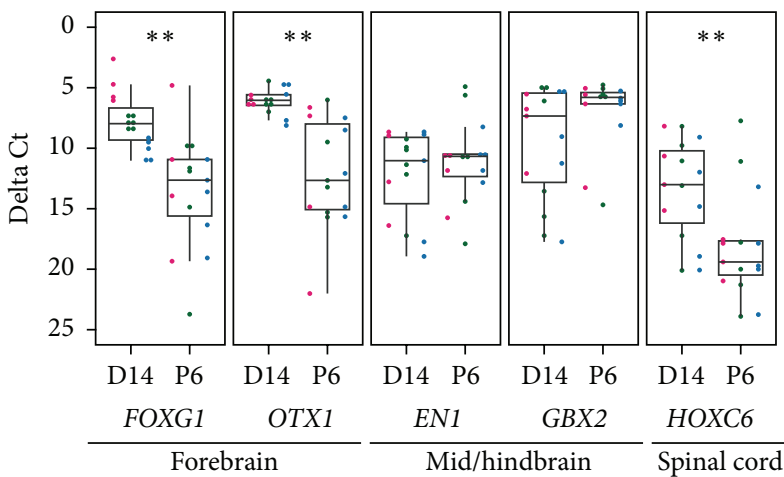

FOXG1 at day 14

- High (4) - Low (5)

- Middle (6)

(c)

FIgURE 4: Continued. 


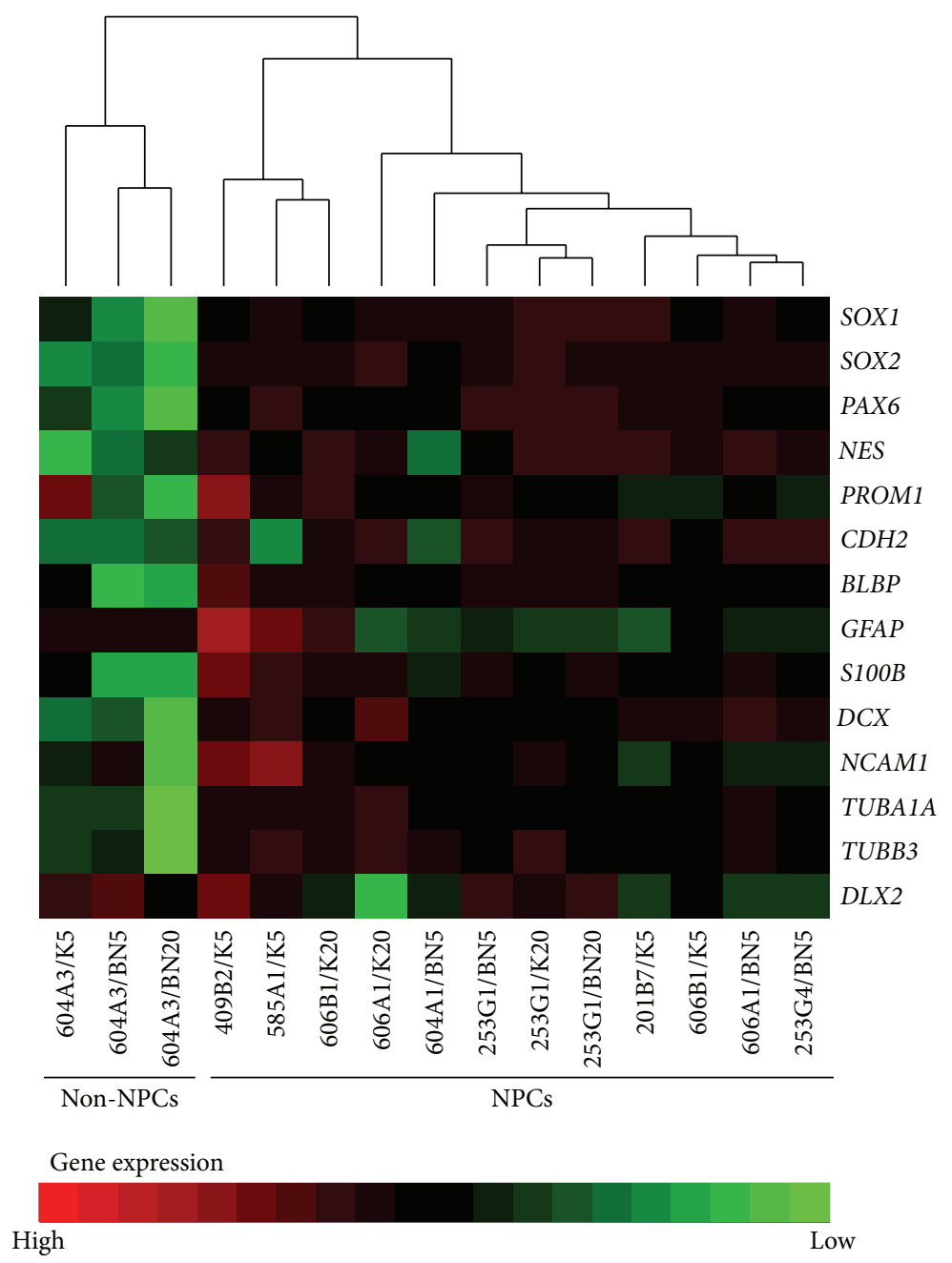

(d)

Figure 4: Established hNPCs express regional markers indicative of a mid/hindbrain-type property. (a) Morphologies of the expanded neurospheres at approximately passage 6 (upper panel). $\beta$ III tubulin-positive neurons after 14 days of neuronal differentiation. Green: $\beta$ III tubulin; blue: nucleus (lower panel). Scale bars: $500 \mu \mathrm{m}$ (black) and $50 \mu \mathrm{m}$ (white). (b) Neurite analysis after 14 days of neuronal differentiation. Relative total neurite length is shown as the mean \pm SD. Statistical analysis was performed using Dunnett's test. ${ }^{*} p<0.05$ and ${ }^{* *} p<0.01$. (c) Dot plots and box plots showing the expression of forebrain markers (FOXG1 and OTX1), mid/hindbrain markers (EN1 and GBX2), and a spinal cord marker (HOXC6) in day 14 (D14) neural aggregates and neurospheres at approximately passage 6 (P6). The numbers of clones analyzed are shown in parentheses. Statistical analysis was performed using Welch's two-sample $t$-test. ${ }^{* *} p<0.01$. (d) Hierarchical clustering of clones based on neural progenitor marker expression. Red: high gene expression; green: low gene expression.

identified by screening for FOXG1 expression. To evaluate this possibility, we grouped all of the neural aggregates into four categories (FOXG1-high/SOX1-high, FOXG1-middle/SOX1high, FOXG1-low/SOX1-high, and FOXG1-low/SOX1-low) by clustering based on FOXG1 and SOX1 expression levels (Figure 3(c) and Table S3). We then selected neural aggregates from each category, except for the SOX1-low, nonneural lineage category, for further expansion. Notably, these neural aggregates included clones derived from each of the three different tissue types (Table S3).

To facilitate the expansion of homogeneous populations of hNPCs, we cultured them as floating neurospheres [22] rather than as adherent cells like lt-NES cells. Expandable neurospheres were established at approximately passage 6 regardless of the FOXG1 expression level in day 14 neural aggregates or the somatic tissue origin (Figure 4(a)). To confirm that the neurospheres were hNPCs, neuronal differentiation was induced using the serum-free neuronal differentiation protocol. All of the HDF- and CB cell-derived neurospheres differentiated into $\beta$ III tubulin-positive neurons with long neurites, whereas only two of the five PBMN cell-derived neurospheres underwent neuronal differentiation (Figures 4(a) and 4(b)). Notably, all of the clones that failed to differentiate were derived from the hiPSC clone 604A3 (Figures 4(a) and 4(b)). These results also indicated that hNPC establishment was independent of the FOXG1 
expression level in day 14 neural aggregates and of the hiPSC somatic tissue origin. FOXG1 expression decreases with increasing passage of lt-NES cells $[9,10]$ and neurospheres [22]. Thus, we hypothesized that FOXG1 expression in day 14 neural aggregates may decrease over time, resulting in the formation of $\mathrm{mid} /$ hindbrain-type progenitors. To assess this supposition, we compared the expression of regional identity marker genes in day 14 neural aggregates and passage 6 neurospheres (Figure 4(c)). We found that the neural aggregates expressed variable levels of not only the forebrain markers, FOXG1 and OTX1, but also each marker gene for the $\mathrm{mid} /$ hindbrain (EN1/GBX2) and spinal cord (HOXC6) (Figure $4(\mathrm{c})$ ). These broad expression patterns of regional marker genes were consistent with a previous report on the activity of endogenous Wnt signaling [34]. In contrast, passage 6 neurospheres expressed higher and less variable levels of the $\mathrm{mid} /$ hindbrain markers and reduced levels of the forebrain and spinal cord markers (Figure 4(c)). Thus, while the day 14 neural aggregates exhibited upregulated forebrain marker expression, established hNPCs displayed a mid/hindbrainlike regional property, consistent with previous findings [22].

Finally, we compared the expression of neural progenitor genes among the established hNPCs (Figure 4(d)). We found that hNPCs and 604A3-derived non-hNPCs were separately clustered and that the hNPCs exhibited similar neural progenitor gene expression patterns regardless of the dSMADi conditions and their somatic tissue origin. All of the PBMN clones met our criteria of high SOX1 and PAX6 expressions on day 14 (Figure 3(c)). All three of the 604A3 clones had almost the same properties on day 14 but differed from the other clones at passage 6 (Figure $4(\mathrm{~d})$ ). We could not eliminate the possibility that the day 14 neural aggregates contained nonneural cells, because gene expression analysis was performed on the bulk population, not at the single-cell level. Therefore, we applied the neurosphere culture method to select a homogeneous population and we were successful in almost every case. However, given that at least neural crest cells can grow in our neurosphere conditions [35], some cell population that was preferentially induced in the 604A3 clone might have been selected and expanded in the neurosphere culture process.

\section{Conclusions}

We conclude that neural lineage cells can be derived from most hiPSC clones, regardless of their somatic tissue origin, using dual SMAD inhibition. We found that PBMN cellderived hiPSC clones did not exhibit increased expression of the forebrain marker gene, FOXG1, but generated hNPCs with neuronal differentiation ability as efficiently as HDFand CB-derived hiPSC clones. Moreover, neural aggregates at the early neural induction stage exhibited variable neural regional marker gene expression patterns and gave rise to hNPCs that uniformly exhibited a mid/hindbrain-type property and expressed similar levels of neural progenitor genes. These findings suggest that the hNPCs described here may be a useful cell source for basic and pharmaceutical research aimed at developing regenerative therapies for treating various neuronal diseases.

\section{Competing Interests}

The authors declare that there are no competimg interests regarding the publication of this paper.

\section{Authors' Contributions}

Yonehiro Kanemura conceived the study and wrote the paper with Hayato Fukusumi and finally approved it. Yonehiro Kanemura, Hayato Fukusumi, Tomoko Shofuda, and Yohei Bamba designed experiments. Hayato Fukusumi, Tomoko Shofuda, Atsuyo Yamamoto, Daisuke Kanematsu, and Yukako Handa collected and analyzed data. Keisuke Okita established and distributed hiPSC clones. Masaya Nakamura, Shinya Yamanaka, and Hideyuki Okano provided critical reading and scientific discussions.

\section{Acknowledgments}

The authors thank Ms. Ai Takada, Miho Sumida, Ema Yoshioka, and Yui Inazawa for their technical support. The authors received generous support from Dr. Kazutoshi Takahashi (CiRA, Kyoto University). This study was supported by the Research Center Network for Realization of Regenerative Medicine of Japan, the Japan Science and Technology Agency (JST), and the Japan Agency for Medical Research and Development (AMED) and was also partially supported by the Research on Regulatory Harmonization and Evaluation of Pharmaceuticals, Medical Devices, Regenerative and Cellular Therapy Products, Gene Therapy Products, and Cosmetics from AMED.

\section{References}

[1] J. A. Thomson, J. Itskovitz-Eldor, S. S. Shapiro et al., "Embryonic stem cell lines derived from human blastocysts," Science, vol. 282, no. 5391, pp. 1145-1147, 1998.

[2] K. Takahashi, K. Tanabe, M. Ohnuki et al., "Induction of pluripotent stem cells from adult human fibroblasts by defined factors," Cell, vol. 131, no. 5, pp. 861-872, 2007.

[3] J. Yu, M. A. Vodyanik, K. Smuga-Otto et al., "Induced pluripotent stem cell lines derived from human somatic cells," Science, vol. 318, no. 5858, pp. 1917-1920, 2007.

[4] K. Okita, T. Yamakawa, Y. Matsumura et al., "An efficient nonviral method to generate integration-free human-induced pluripotent stem cells from cord blood and peripheral blood cells," STEM CELLS, vol. 31, no. 3, pp. 458-466, 2013.

[5] A. Nasu, M. Ikeya, T. Yamamoto et al., "Genetically matched human iPS cells reveal that propensity for cartilage and bone differentiation differs with clones, not cell type of origin," PLoS ONE, vol. 8, no. 1, Article ID e53771, 2013.

[6] K. Osafune, L. Caron, M. Borowiak et al., "Marked differences in differentiation propensity among human embryonic stem cell lines," Nature Biotechnology, vol. 26, no. 3, pp. 313-315, 2008.

[7] S.-C. Zhang, M. Wernig, I. D. Duncan, O. Brüstle, and J. A. Thomson, "In vitro differentiation of transplantable neural precursors from human embryonic stem cells," Nature Biotechnology, vol. 19, no. 12, pp. 1129-1133, 2001.

[8] Y. Elkabetz, G. Panagiotakos, G. Al Shamy, N. D. Socci, V. Tabar, and L. Studer, "Human ES cell-derived neural rosettes reveal 
a functionally distinct early neural stem cell stage," Genes and Development, vol. 22, no. 2, pp. 152-165, 2008.

[9] A. Falk, P. Koch, J. Kesavan et al., "Capture of neuroepitheliallike stem cells from pluripotent stem cells provides a versatile system for in vitro production of human neurons," PLOS ONE, vol. 7, no. 1, Article ID e29597, 2012.

[10] P. Koch, T. Opitz, J. A. Steinbeck, J. Ladewig, and O. Brüstle, "A rosette-type, self-renewing human ES cell-derived neural stem cell with potential for in vitro instruction and synaptic integration," Proceedings of the National Academy of Sciences of the United States of America, vol. 106, no. 9, pp. 3225-3230, 2009.

[11] D.-S. Kim, J. S. Lee, J. W. Leem et al., "Robust enhancement of neural differentiation from human ES and iPS cells regardless of their innate difference in differentiation propensity," Stem Cell Reviews and Reports, vol. 6, no. 2, pp. 270-281, 2010.

[12] T. Wada, M. Honda, I. Minami et al., "Highly efficient differentiation and enrichment of spinal motor neurons derived from human and monkey embryonic stem cells," PLoS ONE, vol. 4, no. 8, Article ID e6722, 2009.

[13] S. M. Chambers, C. A. Fasano, E. P. Papapetrou, M. Tomishima, M. Sadelain, and L. Studer, "Highly efficient neural conversion of human ES and iPS cells by dual inhibition of SMAD signaling," Nature Biotechnology, vol. 27, no. 3, pp. 275-280, 2009.

[14] A. Morizane, D. Doi, T. Kikuchi, K. Nishimura, and J. Takahashi, "Small-molecule inhibitors of bone morphogenic protein and activin/nodal signals promote highly efficient neural induction from human pluripotent stem cells," Journal of Neuroscience Research, vol. 89, no. 2, pp. 117-126, 2011.

[15] K. Watanabe, M. Ueno, D. Kamiya et al., "A ROCK inhibitor permits survival of dissociated human embryonic stem cells," Nature Biotechnology, vol. 25, no. 6, pp. 681-686, 2007.

[16] M. Eiraku, K. Watanabe, M. Matsuo-Takasaki et al., "Selforganized formation of polarized cortical tissues from ESCs and its active manipulation by extrinsic signals," Cell Stem Cell, vol. 3, no. 5, pp. 519-532, 2008.

[17] S. R. Stacpoole, B. Bilican, D. J. Webber et al., "Efficient derivation of NPCs, spinal motor neurons and midbrain dopaminergic neurons from hESCs at 3\% oxygen," Nature Protocols, vol. 6, no. 8, pp. 1229-1240, 2011.

[18] M. Koyanagi-Aoi, M. Ohnuki, K. Takahashi et al., "Differentiation-defective phenotypes revealed by largescale analyses of human pluripotent stem cells," Proceedings of the National Academy of Sciences of the United States of America, vol. 110, no. 51, pp. 20569-20574, 2013.

[19] Y. Imaizumi, Y. Okada, W. Akamatsu et al., "Mitochondrial dysfunction associated with increased oxidative stress and $\alpha$ synuclein accumulation in PARK2 iPSC-derived neurons and postmortem brain tissue," Molecular Brain, vol. 5, article 35, 2012.

[20] E. Ohta, T. Nihira, A. Uchino et al., "I2020T mutant LRRK2 iPSC-derived neurons in the Sagamihara family exhibit increased Tau phosphorylation through the AKT/GSK-3 $\beta$ signaling pathway," Human Molecular Genetics, vol. 24, no. 17, pp. 4879-4900, 2015.

[21] Y. Kanemura, H. Mori, S. Kobayashi et al., "Evaluation of in vitro proliferative activity of human fetal neural stem/progenitor cells using indirect measurements of viable cells based on cellular metabolic activity," Journal of Neuroscience Research, vol. 69, no. 6, pp. 869-879, 2002.

[22] T. Shofuda, H. Fukusumi, D. Kanematsu et al., "A method for efficiently generating neurospheres from human-induced pluripotent stem cells using microsphere arrays," NeuroReport, vol. 24, no. 2, pp. 84-90, 2013.

[23] O. Adewumi, B. Aflatoonian, L. Ahrlund-Richter et al., "Characterization of human embryonic stem cell lines by the International Stem Cell Initiative," Nature Biotechnology, vol. 25, no. 7, pp. 803-816, 2007.

[24] C. A. Schneider, W. S. Rasband, and K. W. Eliceiri, "NIH image to imageJ: 25 years of image analysis," Nature Methods, vol. 9, no. 7, pp. 671-675, 2012.

[25] H. Mori, K. Ninomiya, M. Kino-Oka et al., "Effect of neurosphere size on the growth rate of human neural stem/progenitor cells," Journal of Neuroscience Research, vol. 84, no. 8, pp. 16821691, 2006.

[26] L. Dehmelt, G. Poplawski, E. Hwang, and S. Halpain, "NeuriteQuant: an open source toolkit for high content screens of neuronal Morphogenesis," BMC Neuroscience, vol. 12, article 100, 2011.

[27] R.-H. Xu, R. M. Peck, D. S. Li, X. Feng, T. Ludwig, and J. A. Thomson, "Basic FGF and suppression of BMP signaling sustain undifferentiated proliferation of human ES cells," Nature Methods, vol. 2, no. 3, pp. 185-190, 2005.

[28] K. Watanabe, D. Kamiya, A. Nishiyama et al., "Directed differentiation of telencephalic precursors from embryonic stem cells," Nature Neuroscience, vol. 8, no. 3, pp. 288-296, 2005.

[29] T. A. Blauwkamp, S. Nigam, R. Ardehali, I. L. Weissman, and R. Nusse, "Endogenous Wnt signalling in human embryonic stem cells generates an equilibrium of distinct lineage-specified progenitors," Nature Communications, vol. 3, article 1070, 2012.

[30] K. Kim, A. Doi, B. Wen et al., "Epigenetic memory in induced pluripotent stem cells," Nature, vol. 467, no. 7313, pp. 285-290, 2010.

[31] R. Lister, M. Pelizzola, Y. S. Kida et al., "Hotspots of aberrant epigenomic reprogramming in human induced pluripotent stem cells," Nature, vol. 471, no. 7336, pp. 68-73, 2011.

[32] J. M. Polo, S. Liu, M. E. Figueroa et al., "Cell type of origin influences the molecular and functional properties of mouse induced pluripotent stem cells," Nature Biotechnology, vol. 28, no. 8, pp. 848-855, 2010.

[33] M. N. Manuel, B. Martynoga, M. D. Molinek et al., "The transcription factor Foxg1 regulates telencephalic progenitor proliferation cell autonomously, in part by controlling Pax6 expression levels," Neural Development, vol. 6, article 9, 2011.

[34] N. Moya, J. Cutts, T. Gaasterland, K. Willert, and D. A. Brafman, "Endogenous WNT signaling regulates hPSC-derived neural progenitor cell heterogeneity and specifies their regional identity," Stem Cell Reports, vol. 3, no. 6, pp. 1015-1028, 2014.

[35] G. Lee, S. M. Chambers, M. J. Tomishima, and L. Studer, "Derivation of neural crest cells from human pluripotent stem cells," Nature Protocols, vol. 5, no. 4, pp. 688-701, 2010. 

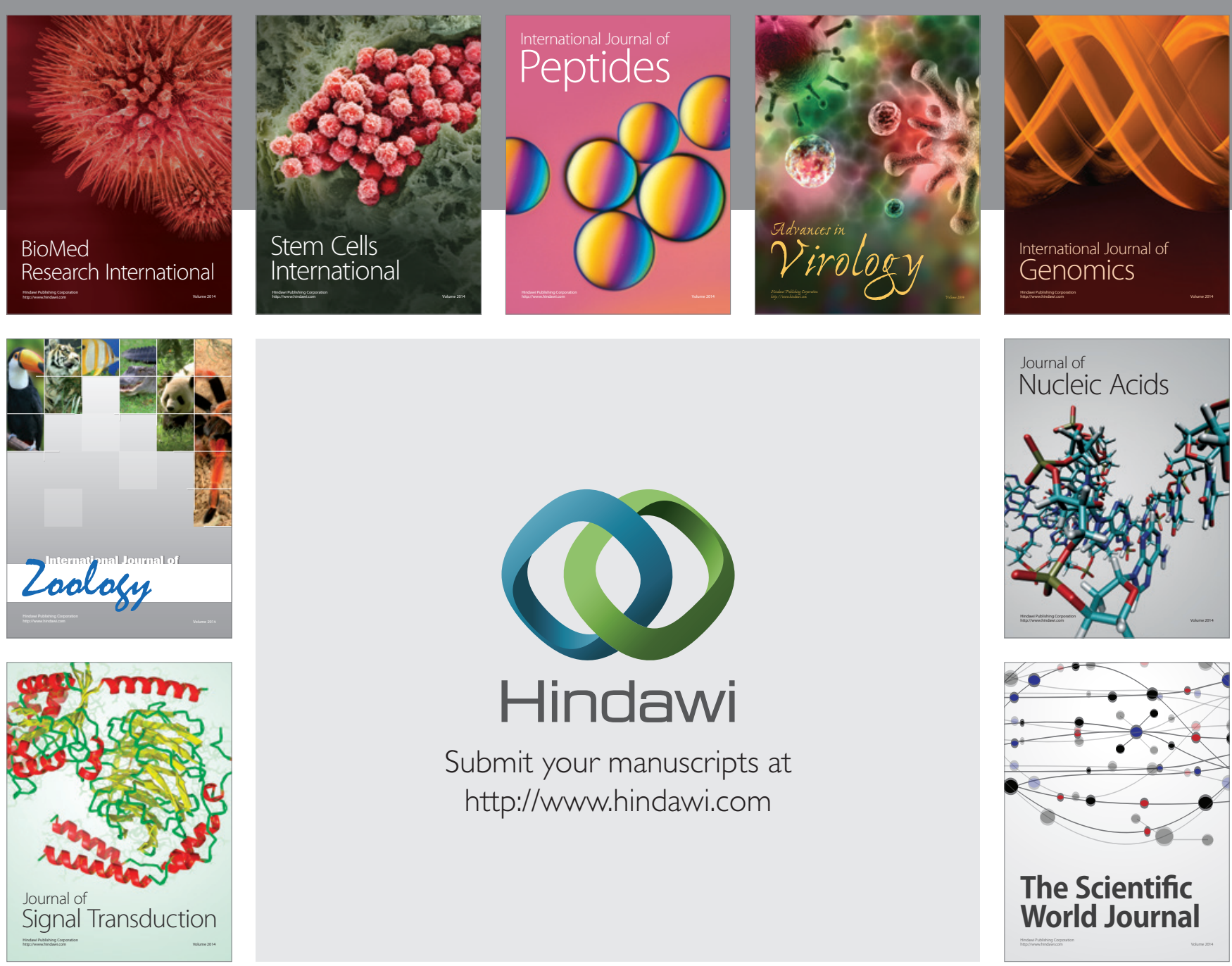

Submit your manuscripts at

http://www.hindawi.com
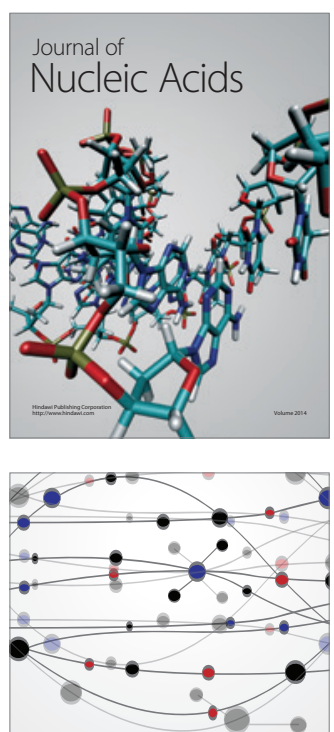

The Scientific World Journal
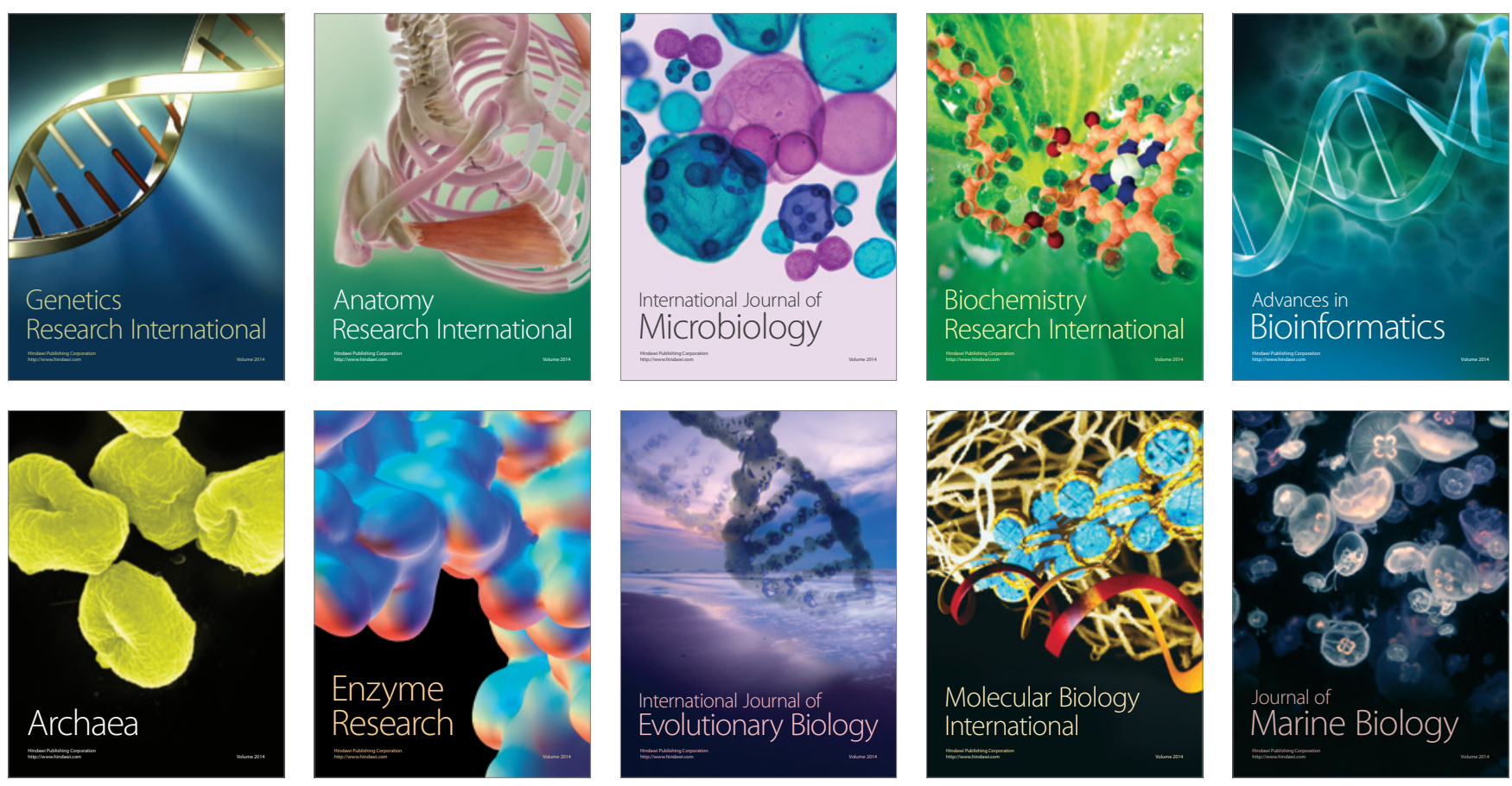